\title{
CALCULATION OF TUMBLING BOUNDARIES OF A GENERIC WING-ONLY AIRLINER
}

\author{
A. Khrabrov and M. Sidoryuk \\ Central Aerohydrodynamic Institute (TsAGI) \\ 1 Zhukovsky Str., Zhukovsky 140180, Moscow Region, Russia
}

\begin{abstract}
The aerodynamic model of a generic wing-only airliner configuration is developed for a whole range of angles of attack $\left(-180^{\circ} \ldots+180^{\circ}\right)$, based on experimental data obtained in wind tunnels using static, and forced oscillations. Two different approaches for the tumbling boundaries calculation are used. In the first approach, the steady pitch autorotation is calculated. In the second approach, for various angles of attack, the minimum pitch rate disturbance is considered which can result in tumbling. The tumbling boundaries are calculated via the use of a continuation technique. The dependence of these boundaries on such parameters as flight altitude, aircraft center of gravity position, and total velocity is analyzed.
\end{abstract}

\section{NOMENCLATURE}

\author{
c.g. center of gravity \\ $\bar{c} \quad$ mean chord length \\ $C_{m} \quad$ aircraft pitching-moment coefficient about the center of gravity \\ $C_{X} \quad$ aircraft tangential force coefficient \\ $C_{Z} \quad$ aircraft normal force coefficient \\ $I_{y y} \quad$ aircraft moment of inertia about the pitch axis \\ $q \quad$ pitch rate \\ $S \quad$ wing area \\ $V \quad$ total velocity \\ $\alpha \quad$ angle of attack \\ $\theta \quad$ pitch angle
}

\section{INTRODUCTION}

It is well known that flying wing aircraft configurations are susceptible to a flight instability called tumbling. Tumbling is an autorotative pitching motion, pri-

This is an Open Access article distributed under the terms of the Creative Commons Attribution License 4.0, which permits unrestricted use, distribution, and reproduction in any medium, provided the original work is properly cited. 
marily about an axis parallel to the aircraft's lateral axis, combined with planar translation. The renewed interest in the study of tumbling is caused by the current development of wing-only aircraft configurations. The well-known examples are the concept of a future high-capacity blended-wing-body (BWB) subsonic transport airplane [1] and various tailless unmanned aerial vehicles. It is difficult, if not impossible, to escape the tumbling motion once it develops. To prevent the onset of tumbling, it is important to understand the causes of this dangerous phenomenon and to know its borders. The experimental investigations showed that the tumbling modes are very sensitive to geometric and inertial characteristics of the aircraft [2]. The initial conditions also essentially influence the tumbling motion. The effect of the geometry and mass distribution on tumbling sensitivity must be taken into account by aircraft designers.

The effects of initial conditions, degrees of freedom, Reynolds number, and aircraft static margin on the tumbling characteristics of a wing-only aircraft were investigated in [3] using the approach of simultaneous solution of flight dynamics and computational fluid dynamics equations. However, direct simulation with some restricted set of initial conditions cannot give a complete picture of tumbling boundaries and its parameters. The purpose of the present research is the nonlinear mathematical model development and qualitative analysis of tumbling boundaries for a generic wing-only aircraft. The study of tumbling requires knowledge of aerodynamic characteristics in the whole range of angles of attack from $-180^{\circ}$ to $+180^{\circ}$. Aerodynamic characteristics have substantial asymmetry at high angles of attack. So, generally speaking, the tumbling phenomenon should be investigated based on a full model of aircraft spatial motion. In this paper, the tumbling boundaries are calculated based on the longitudinal motion analysis only. Main features of the tumbling dynamics are captured by this consideration. In the paper, the qualitative methods of nonlinear dynamics with a continuation technique [4-6] as a main tool are used.

Two different tumbling modes are considered: a steady-state autorotation and a tumbling onset, i. e., a minimal deviation of initial conditions from a level flight which results in tumbling. These problem statements lead to the two different parameter dependent boundary-value problems. Both are solved numerically via continuation on the parameter. The principal continuation parameter is the elevator deflection. Influence of other parameters such as aircraft center of gravity position and flight altitude is investigated.

\section{AERODYNAMIC MODEL}

The mathematical model of a generic flying wing aircraft configuration was developed using various experimental data and results of Reynolds-averaged NavierStokes (RANS) calculations in the whole range of angle of attack. Figure 1 shows nondimensional tangential and normal forces and pitching moment de- 


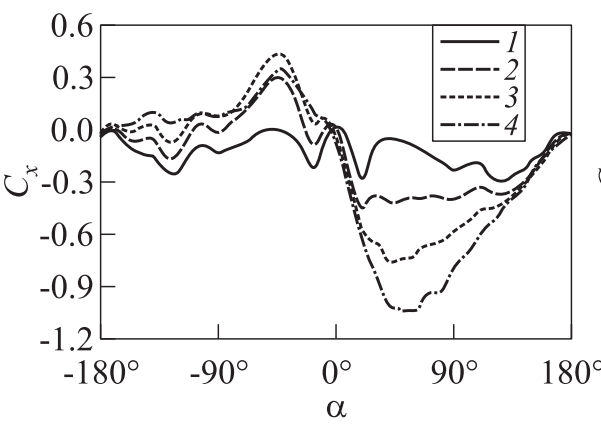

(a)

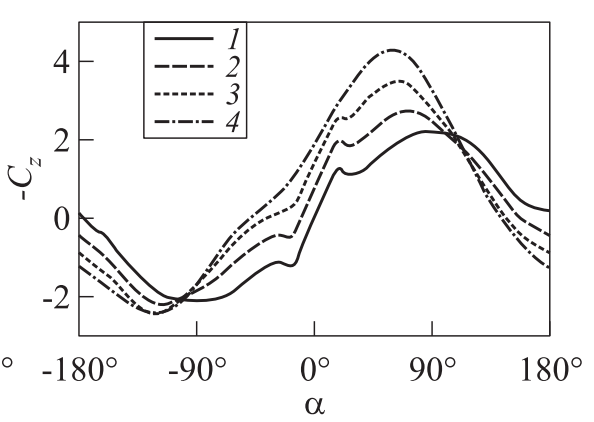

(b)

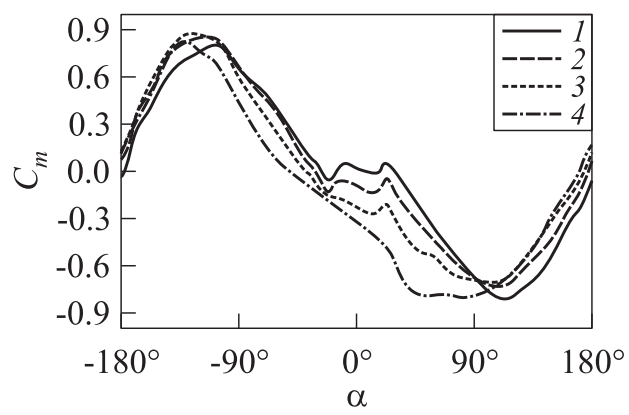

(c)

Figure 1 Static (1) and dynamic contributions to the aircraft tangential force $(a)$, normal force $(b)$, and pitching moment for $x_{\text {c.g. }} / \bar{c}=0.275: 2-$ nondimensional pitch rate $=0.2 ; 3-0.4 ;$ and $4-$ nondimensional pitch rate $=0.6$

pending on angle of attack in the range $-180^{\circ}<\alpha<+180^{\circ}$ for static condition and for several values of nondimensional pitch rate. The aerodynamic characteristics are highly nonlinear and dependent on the pitch rate and pitch direction. Mass/inertia and geometric parameters of the considered generic wing-only aircraft model are the following: mass $m=70370 \mathrm{~kg}$; moments of inertia about the roll, pitch, and yaw axis $I_{x x}=4597000 \mathrm{~kg} \cdot \mathrm{m}^{2}, I_{y y}=5902000 \mathrm{~kg} \cdot \mathrm{m}^{2}$, and $I_{z z}=5126000 \mathrm{~kg} \cdot \mathrm{m}^{2}$, respectively; $\bar{c}=8.00 \mathrm{~m}$; and $S=373.5 \mathrm{~m}^{2}$.

\section{CALCULATION OF TUMBLING}

\subsection{Longitudinal Motion}

To study the phenomenon of tumbling, the longitudinal motion of aircraft is considered. It is described by the following forth-order system of equations: 


$$
\left.\begin{array}{rl}
\frac{d u}{d t} & =-q w+\frac{\rho V^{2} S}{2 m} C_{X}-g \sin \theta+\frac{T}{m} ; \\
\frac{d w}{d t} & =q u+\frac{\rho V^{2} S}{2 m} C_{Z}+g \cos \theta ; \\
\frac{d \theta}{d t} & =q ; \\
\frac{d q}{d t} & =\frac{\rho V^{2} S \bar{c}}{2 I_{y y}} C_{m}
\end{array}\right\}
$$

where $[u, w, q, \theta]$ is the standard vector of longitudinal variables consisting of the tangential and normal velocity components, pitch rate, and pitch angle; $C_{X}(\alpha, \bar{q}), C_{Z}(\alpha, \bar{q})$, and $C_{m}\left(\alpha, \bar{q}, \delta_{e}\right)$ are the dimensionless aerodynamic coefficients of tangential and normal forces and the body-axis pitch moment; $\bar{q}=q \bar{c} /(2 V)$ is the dimensionless pitch rate; and $\delta_{e}$ and $T$ are the elevator deflection, and thrust force, respectively.

\section{$3.2 \quad$ Numerical Tools}

Main methods of analysis of many nonlinear dynamic phenomena are qualitative and numerical methods for nonlinear dynamical systems [4-7]. Numerical investigation is carried out by continuation of stationary and periodic (rotational) solutions of nonlinear system with simultaneous calculation of their stability. For searching for rotational periodic solutions, it is convenient to write system (1) as follows:

$$
\frac{d x}{d t}=F(x, \delta)
$$

where $x=[u, w, q, \theta]$. Periodic rotations of system (2) corresponding to tumbling motion can be described by one of the two following boundary-value problems:

$$
\begin{aligned}
& x(T, \delta)-x(0, \delta)+2 \pi\left[\begin{array}{llll}
0 & 0 & 1 & 0
\end{array}\right]^{\prime}=0 \\
& x(T, \delta)-x(0, \delta)-2 \pi\left[\begin{array}{llll}
0 & 0 & 1 & 0
\end{array}\right]^{\prime}=0
\end{aligned}
$$

where $\delta$ is the continuation parameter and unknown time $T$ is the period of autorotation. Since unknown initial point $x(0)$ of a periodic rotation can be taken arbitrary in the trajectory, one component of vector $x(0)$ must be fixed within the range of its change. It is convenient to take $w(0)=0$, because in this case, the initial angle of attack is also zero: $\alpha(0)=0$. So, only three components of the 4-dimensional vector $x(0)$ are unknown, and Eq. (3) (or Eq. (4)) can be considered as a curve in a 5-dimensional space which includes these 3 components of initial condition, $T$, and $\delta$. In this study, the elevator deflection $\delta_{e}$ is used as the principal continuation parameter, while all other parameters such as flight altitude or aircraft center of gravity position, etc. also vary, but remain 

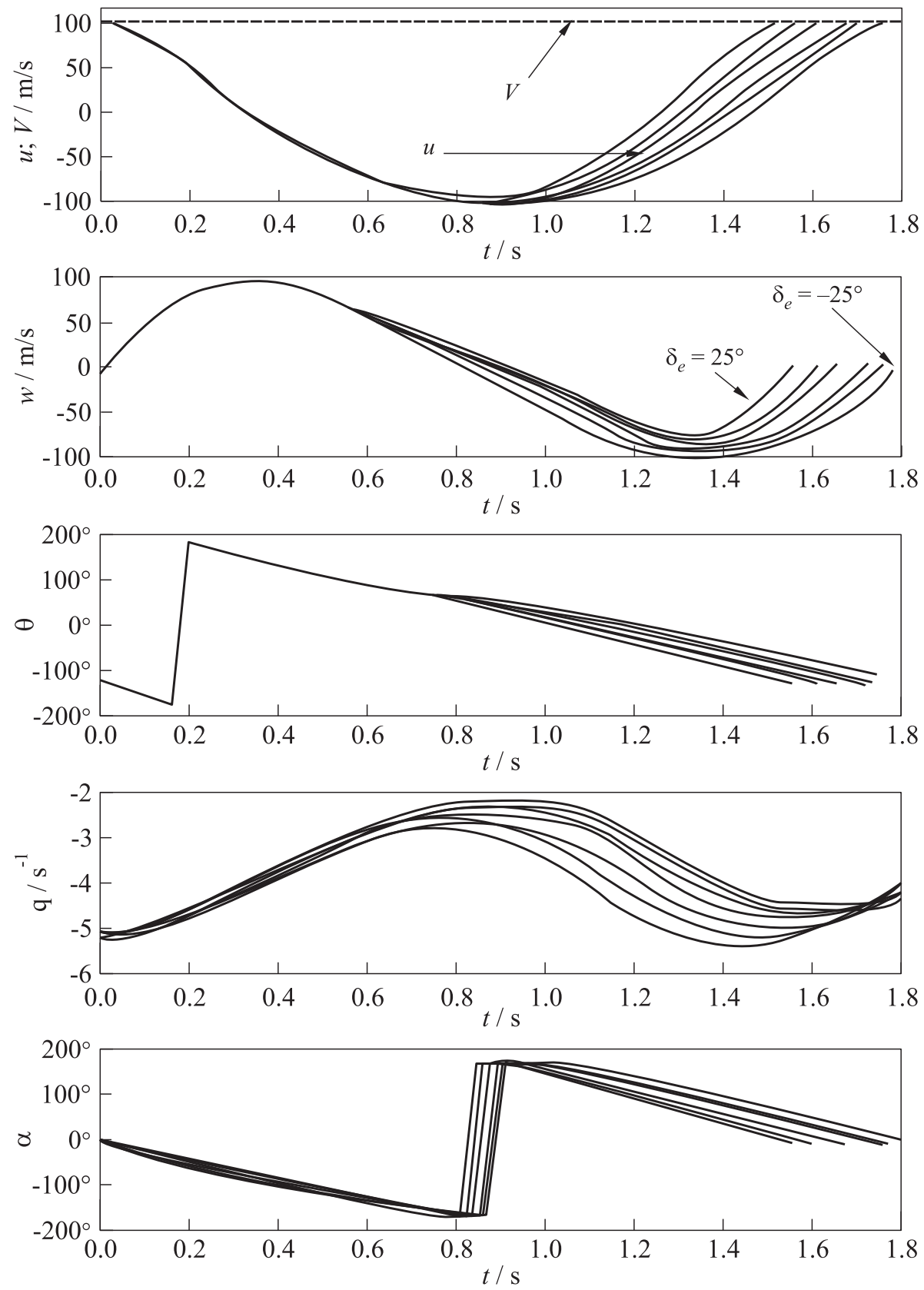

Figure 2 Periodic rotation trajectories depending on elevator deflection, $q<0$ 
fixed during the continuation procedure. For the application of the continuation method, it is necessary to know some starting point on the curve. In this study, a random search of such points is used.

\subsection{Results for the Study of Tumbling}

Figure 2 shows the trajectories of periodic rotations, describing stationary tumbling solutions in system (1) for different elevator deflections in the range $-25^{\circ}$ $<\delta_{e}<25^{\circ}$ with step $4^{\circ}$ for the following parameters: flight altitude $H$ $=12000 \mathrm{~m}$, aircraft center of gravity displacement $\Delta x / \bar{c}=-0.05$ relative the basic value $x / \bar{c}=0.275$, thrust value $T=43.1 \mathrm{kN}$. The thrust value corresponds to the level flight at this altitude with the total velocity $V=100 \mathrm{~m} / \mathrm{s}$. Figure 3 shows maximum and minimum values of these rotations and their period $T$ depending on the elevator deflection. This figure shows also the similar results for center of gravity displacement $\Delta x / \bar{c}=-0.02$. The calculated rotations are stable only in the range of elevator deflections: $18^{\circ}-25^{\circ}$ at $\Delta x / \bar{c}=-0.05$ and $24^{\circ}-25^{\circ}$ at $\Delta x / \bar{c}=-0.02$ (they are shown by large markers).
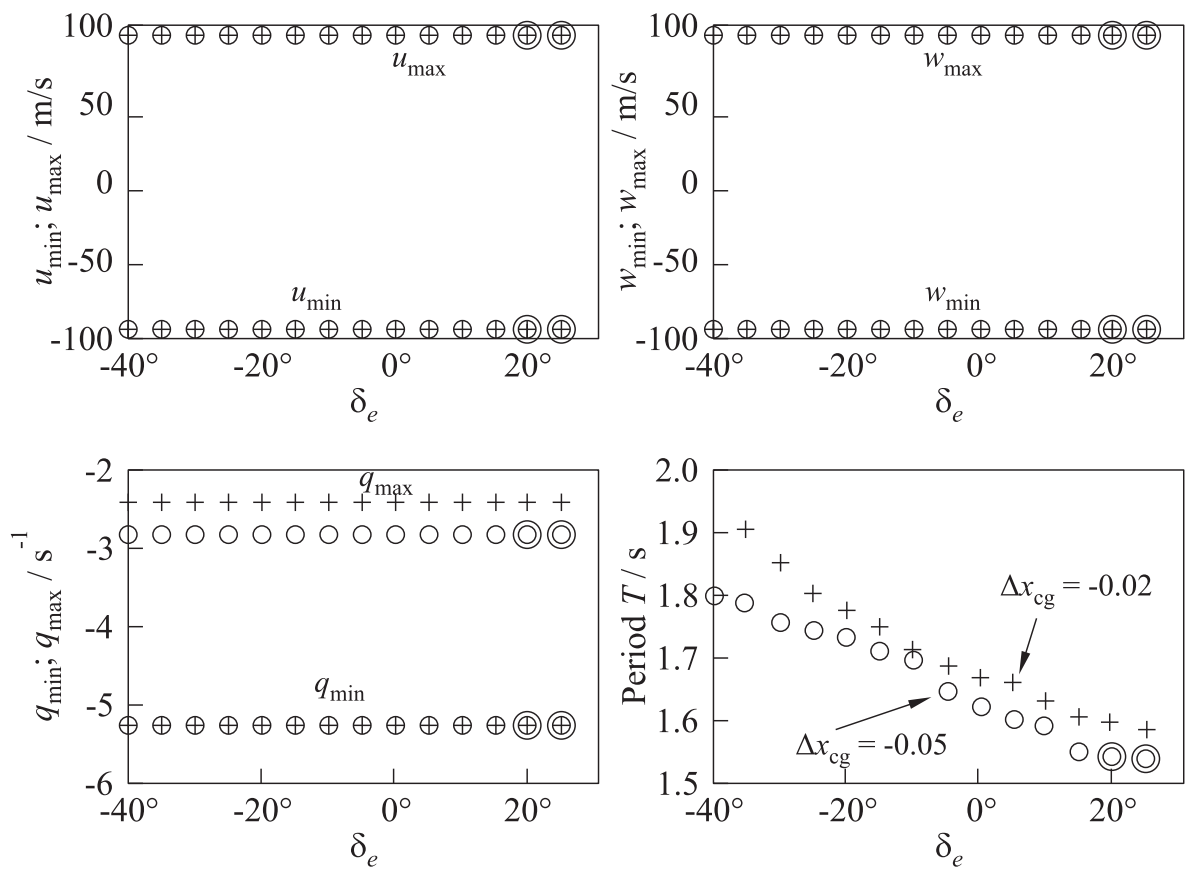

Figure 3 Maximum and minimum values of periodic rotation trajectories and their period depending on elevator deflection, $q<0$ 

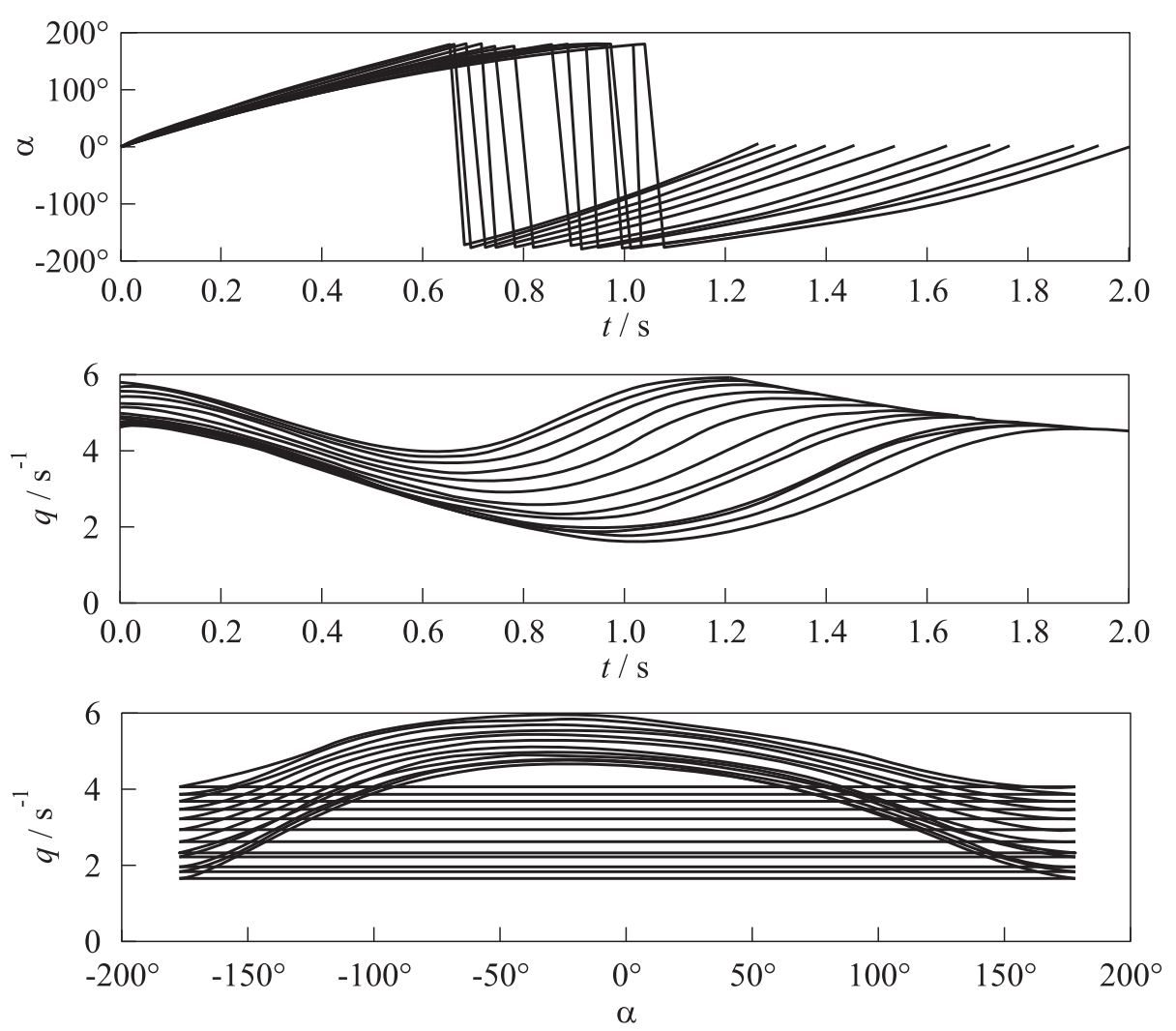

Figure 4 Periodic rotation trajectories depending on elevator deflection, $q>0$

The parameter dependent boundary-value problem (4) has the similar solutions corresponding to rotations with pitch rate $q<0$. Figure 4 shows $\alpha$ and $q$ components of this solutions for the same grid in the parameter $\delta_{e}$ and the same flight parameters as in Fig. 2. All these periodic rotations are unstable.

The fact of instability of periodic solutions at most physically admissible parameters means that the considered aircraft has no tendency to tumbling. Nevertheless, large disturbances can lead to one or several aircraft turns. It is important to calculate the boundaries of such tumbling motion, i. e., to find the initial conditions for which at least one turn occurs. For estimation of the domains of tumbling, note that total velocity is practically constant during a turn. This allows considering this problem for a short-period approximation of the longitudinal motion.

To determine the parameters of tumbling onset, a grid in $(\alpha-q)$ plane with a sufficiently small step was used. Nodes of this grid were used as initial condi- 


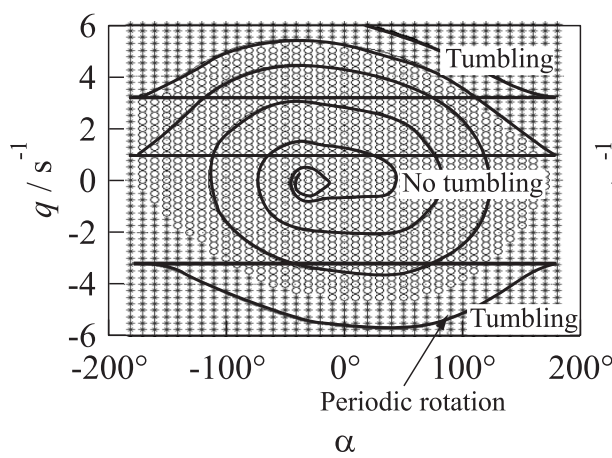

(a)

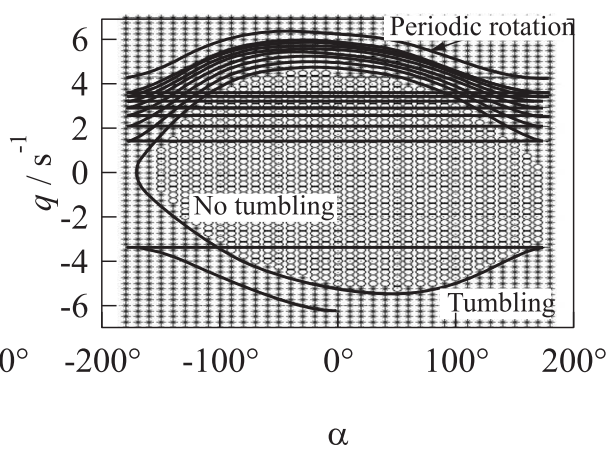

(b)

Figure 5 Domains of tumbling, stable periodic rotation, and example of trajectory originated in the region of tumbling at $H=12 \mathrm{~km} ; V=100 \mathrm{~m} / \mathrm{s} ; \Delta x / \bar{c}=-0.05$; and $\delta=0^{\circ}(a)$ and $-30^{\circ}(b)$

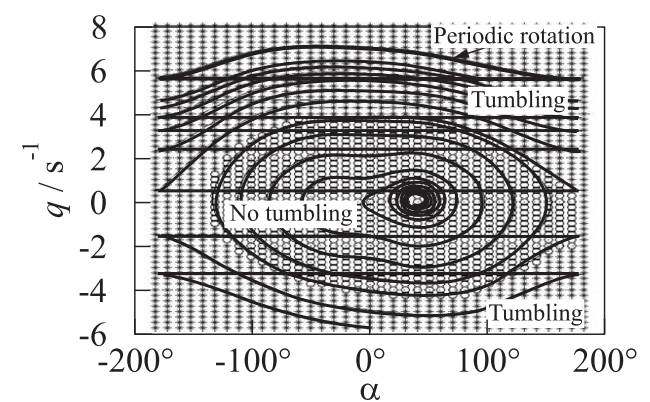

Figure 6 Domains of tumbling, stable periodic rotation, and example of trajectory originated in the region of tumbling at $H=12 \mathrm{~km}$; $V=100 \mathrm{~m} / \mathrm{s} ; \Delta x / \bar{c}=-0.1 ;$ and $\delta=-30^{\circ}$ tions for simulation of the shortperiod approximation of system (1). If the outgoing trajectory reaches angle of attack equal to $+180^{\circ}$ or $-180^{\circ}$ at least once, the point is marked as belonging to the tumbling domain. Otherwise, there is no tumbling. Figures 5 and 6 show the areas of tumbling for several values of the parameters: center of gravity position and elevator deflection. Several trajectories including trajectories of periodic rotations are also shown in these figures.

Numerical simulation shows that regions tumbling absence in the $(\alpha-q)$ plane are convex. This allows to formulate the tumbling onset problem as follows: for each $\alpha$ value, find such a minimal (in absolute value) $q$ that the boundary-value problem

$$
\alpha(T)=\pi
$$

has a solution and find such a minimal $q$ that the boundary-value problem

$$
\alpha(T)=-\pi
$$

has a solution. The above problem formulation allows calculating the tumbling boundaries at a lower cost than the direct scanning. The results of application 


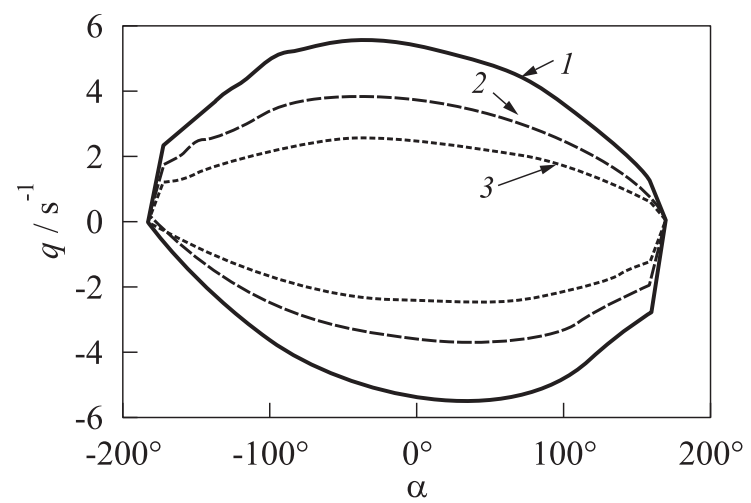

Figure 7 Tumbling boundaries depending on total velocity at $H=12 \mathrm{~km} ; \Delta x / \bar{c}$ $=-0.05 ;$ and $\delta=0^{\circ}: 1-V=110 \mathrm{~m} / \mathrm{s} ; 2-75 ;$ and $3-V=50 \mathrm{~m} / \mathrm{s}$
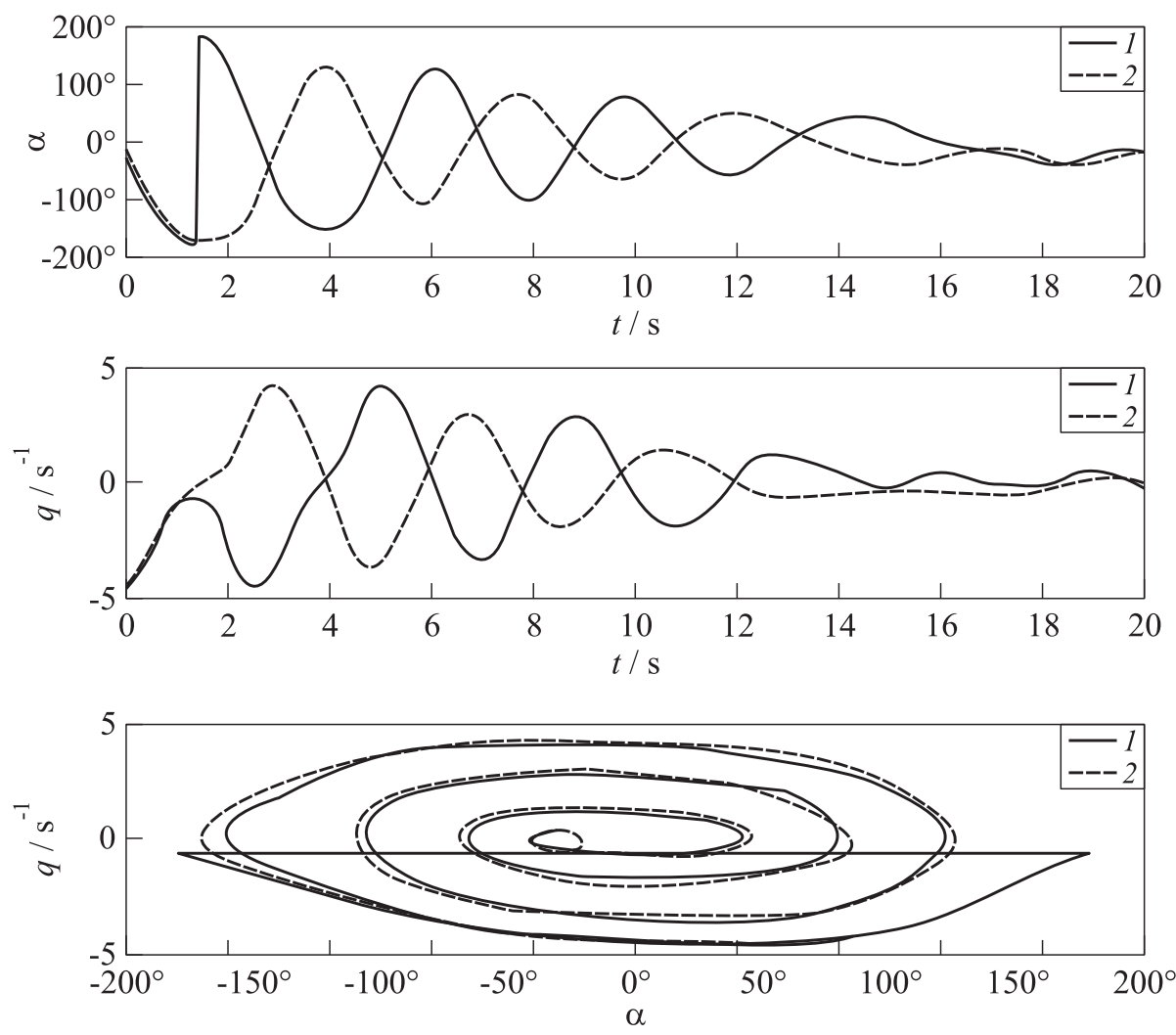

Figure 8 Trajectories near the tumbling boundary: $1-q(0)=0.87 q_{0 \text { circle; }}$ and $2-$ $q(0)=0.86 q_{0}$ circle 
of this algorithm of computing the tumbling boundaries are shown in Fig. 7 . Trajectories near the calculated tumbling boundary are illustrated in Fig. 8. Calculation of tumbling boundaries for the different aircraft and flight parameters has shown that the tumbling boundary moves to the less pitch rates with increasing the flight altitude, or decreasing the total velocity, or increasing forward the aircraft center of gravity position.

\section{CONCLUDING REMARKS}

The problem of the tumbling boundaries of a generic wing-only aircraft has been considered. With the use of continuation technique, periodic autorotation solutions have been calculated. The influence of flight altitude, total velocity, center of gravity position, elevator displacement, and initial conditions has been analyzed. The investigation has shown that the minimum initial pitch rate resulting in tumbling motion decreases with of flight altitude increasing, total velocity decreasing, center of gravity position forward displacement, and elevator positive deflection.

\section{ACKNOWLEDGMENTS}

This work is supported by the Ministry of Education and Science of the Russian Federation within the federal target program "Scientific and scientificpedagogical personnel of innovative Russia" in 2009-2013 (Nos. 14.U01.21.8377 and 14.U01.21.8759) and the Russian Foundation for Basic Research (No. 12-0800679).

\section{REFERENCES}

1. Liebeck, R. H. 2004. Design of the blended wing body subsonic transport. J. Aircraft 41(1):10-25.

2. Fremaux, C. M., D. M. Vairo, and R. D. Whipple. 1995. Effect of geometry and mass distribution on tumbling characteristics of flying wings. J. Aircraft 32(2):404-410.

3. Saephan, S., and C. P. van Dam. 2008. Determination of wing-only aircraft tumbling characteristics through computational fluid dynamics. J. Aircraft 45(3):1044-1054.

4. Zagaynov, G.I., and M. G. Goman. 1984. Bifurcation analysis of critical aircraft flight regimes. ICAS. Toulouse, France. Paper No. 84-4.2.1.

5. Goman, M. G., G.I. Zagaynov, and A.V. Khramtsovsky. 1997. Application of bifurcation methods to nonlinear flight dynamics problems. Prog. Aerosp. Sci. 33(59):539-586.

6. Goman, M. G., and A. V. Khramtsovsky. 1998. Application of continuation and bifurcation methods to the design of control systems. Philos. Trans. Roy. Soc. A 356(1745):2277-2295.

7. Guicheteau, P. 1998. Bifurcation theory: A tool for nonlinear flight dynamics. Philos. Trans. Roy. Soc. A 356:2181-2201. 